\title{
Sistema de mediación familiar en Chile: Reflexiones sobre el acceso a la justicia y la resolución colaborativa ${ }^{3}$
}

\author{
Family Mediation System in Chile: Reflections on Access to Justice and Collaborative Resolution \\ Sistema de mediação familiar no Chile: Reflexóes sobre o acesso à justiça e a resolução colaborativa
}

\section{Resumen}

El presente artículo expondrá los principales resultados alcanzados en el estudio realizado durante el año 2015, que se ocupó de analizar los procesos de mediación familiar en Chile, desde la perspectiva de los mediadores y su valoración en torno al acceso a la justicia y la resolución colaborativa, a partir de la aplicación de modelos y herramientas modificados desde el nuevo sistema de mediación familiar licitada. Para ello, se ha organizado esta exposición en torno a la presentación del problema, el diseño metodológico que guió el estudio, las conclusiones y las propuestas a la luz del trabajo realizado.

Palabras clave: Mediación familiar, acceso a la justicia, mediadores, resolución colaborativa.

\section{Recibido: 16 de marzo, evaluado: 8 de mayo, aprobado: 12 de mayo}

1 Trabajadora social de la Pontificia Universidad Católica de Valparaíso. Magíster en Gestión Colaborativa de Conflictos, Universidad Central de Chile y maestra en Mediación y Negociación por APEP-IUKB en asociación con la Universidad de Ginebra. Especialista en temas de familia, gestión de conflictos y políiticas públicas. Docente e investigadora, Universidad Central de Chile. Correo electrónico: cvaldevenitol@ucentral.cl

2 Trabajadora Social de la Universidad de Chile. Especialista en temas de familia, gestión de conflictos y políticas públicas. Docente e investigadora, Universidad Alberto Hurtado. Correo electrónico: donpaz@gmail.com.

3 Este artículo presenta los resultados de la tesis Mediación Familiar en Chile: Un estudio sobre modelos y técnicas, para optar al grado de Maestra en Mediación y Negociación de APEP-IUKB en asociación con la Universidad de Ginebra, de la estudiante Caterine Valdebenito y la profesora guía María de La Paz Donoso Díaz. 


\begin{abstract}
This article shows the main results obtained in a study carried out in 2015. This study analyzed the processes of family mediation in Chile from the perspective of mediators and their assessment of access to justice and collaborative resolution and from the application of modified models and tools from the newly tendered family mediation system. For this, the present exhibition has been organized in relation to the presentation of the problem, the methodological design that guided the study, the conclusions and the proposals in the work carried out.
\end{abstract}

Keywords: Family mediation, access to justice, mediators, collaborative resolution.

\title{
Resumo
}

O presente artigo exporá os principais resultados atingidos no estudo realizado durante o ano de 2015, que se ocupou em analisar os processos de mediação familiar no Chile, desde a perspectiva dos mediadores e sua valoração em torno do acesso à justiça e a resolução colaborativa, a partir do aplicativo de modelos e ferramentas modificadas desde o novo sistema de mediação familiar adjudicada. Para isto, se organizou esta exposição em relação à apresentação do problema, o desenho metodológico que guiou o estudo, as conclusões e as propostas à luz do trabalho realizado.

Palavras-chave: Mediação familiar, acesso à justiça, mediadores, resolução colaborativa. 


\section{Presentación del tema de investigación}

El cambio del sistema de justicia en temas de familia, en el año 2005, creó un sistema de atención de mediación destinado a apoyar el acceso a justicia con criterios de equidad y calidad, que favoreciese la atención de la población a nivel nacional, que fuese previo al ingreso al sistema judicial tradicional y que privilegiase la resolución por vía colaborativa y de autocomposición entre los intervinientes. Con el paso de los años, el sistema de mediación familiar licitada ${ }^{4}$ avanzó en cobertura a nivel nacional y ha ido modificando sus procedimientos en vistas a mejorar en criterios de calidad de servicio que permitan el progreso de las prestaciones que reciben los y las chilenas en el sistema judicial.

Hoy en día, el SNM organiza su acción en torno a cinco pilares (véase figura 1): la administración del Ministerio de Justicia a través de una unidad especializada como es la Unidad de Mediación ${ }^{5}$ (M. Justicia- UMED, 2014); la administración del registro de mediadores a nivel nacional; la habilitación de los y las profesionales mediadores en las distintas regiones del país, mediante el establecimiento de un conjunto de requisitos; las licitaciones públicas periódicas a través de las que contrata los servicios de centros de mediación desconcentrados a nivel nacional; la atención sin costo para las y los usuarios que presentan causas en los temas previos de mayor concentración de demandas como son pensión de alimentos, cuidado personal y relación directa y regular del hijo/a que no vive con el padre o madre solicitante (Ley 20.260, 2008).
Figura 1. Cinco pilares de acción del SNM

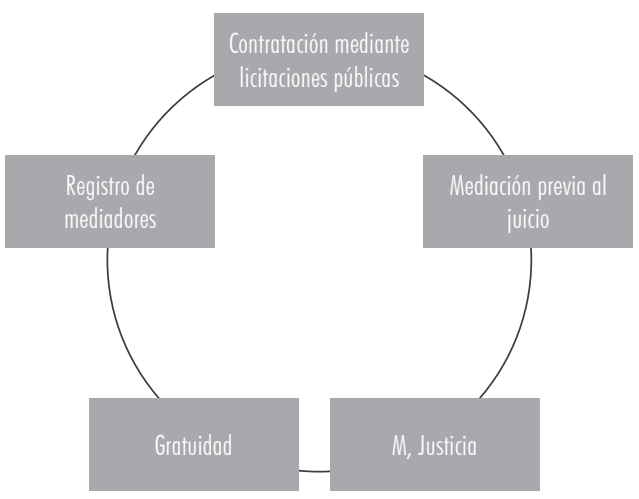

Fuente: UMED, 2014.

En este escenario, la UMED, desde el año 2011 en adelante, se ha ocupado de mirar los procesos de mediación familiar, incorporando la mirada de calidad de servicio ${ }^{6}$, mediante la aplicación de un instrumento creado por la CEAL-PUC (PUCV, 2012), llamado pauta Ecame ${ }^{7}$, destinado a evaluar los desempeños de las y los mediadores que mantienen contratos vigentes con el Estado. Sin embargo, ha estado ausente en este quehacer el levantamiento de las estrategias y elementos que utilizan estos profesionales en sus prácticas laborales, a partir de los cuales sea posible conocer los ejes priorizados, que en diez años de funcionamiento del sistema ha permitido alcanzar y mantener estándares de resultados, de acuerdos y de calidad, adecuados y exitosos, tanto por el sistema de justicia en general y por la UMED; como por las y los usuarios en particular.

En este esquema surge como pregunta guía de la investigación: ¿Cuáles son las técnicas y modelos de la mediación que utilizan los y las mediadores

4 En adelante SNM.

5 En adelante uMED.

6 El concepto de calidad de servicio que pone el Estado está orientado a evaluar que la atención directa del que mediador a las personas sea coherente con los criterios técnicos descritos en las bases de licitación y los instructivos técnicos que regulan el sistema de mediación licitada y que administra el M. de Justicia.

7 El instrumento en cuestión es la Pauta de Evaluación de la Calidad de la Mediación Familiar, diseñado por el Centro de Estudios de la Pontificia Universidad Católica de Valparaíso a petición del M. de Justicia, y ha sido aplicado a nivel nacional en el año 2013 por la Universidad Central de Chile para la evaluación de procesos de atención en mediación familiar. 
familiares en Chile, que les permiten gestionar con éxito en conjunto con las personas los conflictos que abordan? A la base de esta interrogante, se plantearon dos supuestos básicos que es necesario explicar:

a. Que los y las mediadores que operan en el sistema de mediación familiar licitado disponen de herramientas y técnicas propias de la mediación para la gestión y tratamiento del conflicto, en sus fases de apertura, deconstrucción, reordenamiento, búsqueda de soluciones y acuerdos y que son aplicadas con criterios de calidad de servicio.

b. Que los y las usuarios que participan en la mediación familiar presentan problemáticas que corresponden a temas de familia y tienen interés genuino en buscar soluciones a los mismos.

\section{Breve descripción del diseño metodológico utilizado}

Con el planteamiento de la pregunta de investigación y la explícita mención de los supuestos de acción a la base, fue necesario ocuparse del diseño de la investigación que se realizaría, para lo que se comenzó con la definición de los objetivos de la misma. Así surgió como objetivo general de la investigación el analizar la correspondencia entre los modelos, metodologías y las técnicas que aplican los y las mediadores familiares durante el proceso de mediación en Chile que son considerados exitosos y la metodología de calidad de la mediación familiar. Este objetivo fue desagregado en cuatro objetivos específicos que se mencionan a continuación:

a. Distinguir la tipología de modelos que utilizan los y las mediadores que operan en temas de mediación familiar en los distintos momentos del proceso de trabajo.

b. Identificar las herramientas y técnicas que usan los y las mediadores en sus procesos de atención de mediación familiar.

c. Describir los resultados que declaran los y las mediadores sobre el uso de las distintas técnicas aplicadas en el trabajo de mediación realizado. d. Comparar los criterios estandarizados de calidad del proceso de mediación familiar que presenta la pauta Ecame y la percepción de resultados que presentan los operadores del sistema de mediación familiar licitado.

El marco epistemológico que guio esta investigación, y que se enmarcó dentro de las ciencias sociales y de gestión, corresponde al construccionismo, que en palabras de Gergen (2008) es a través de una apreciación crítica del lenguaje como podemos alcanzar la comprensión de nuestras formas de relación con la cultura y, a través de él, abrir un espacio a la consideración de las alternativas futuras de intervención y comprensión de estas prácticas. Esta forma de entender la realidad social fue adecuada para abordar la temática de las prácticas de la mediación vistas y valoradas por los propios actores, ya que permitió recoger, desde la propia vivencia específica de cada uno, la evaluación crítica que realiza de ella; la elaboración de un lenguaje común que favoreció el relato de la experiencia, y así un escenario compartido, el cual aportó en la construcción de una realidad grupal que posibilitó la responsabilidad en el actuar profesional en un esquema normado por la institucionalidad pública. Lo que se entiende acorde con la concepción de la comunicación como categoría social que aporta en el proceso de diferenciación (Pignuoli, 2015) y también de encuentro de los sujetos.

En este marco epistemológico, la investigación que se efectuó fue de corte cualitativo (Flick, 2007) con el propósito de comprender el fenómeno o acontecimiento en estudio desde el interior de mismo, en este caso, desde los procesos generados y relatados por los mismos operadores y operadoras. Por esto, se trabajó desde la óptica del diseño descriptivo e interpretativo de investigación (Taylor \& Bodgman, 1986). La cualidad descriptiva tiene como finalidad definir, clasificar, catalogar o caracterizar el objeto de estudio; en este trabajo, esta cualidad estuvo dada por la importancia de poder recoger y presentar la información de campo, manteniendo su integridad para así avanzar en la representación de la realidad de manera fidedigna. La condición interpretativa se 
asoció con la perspectiva fenomenológica propia de esta metodología, que posibilita que la investigadora experimente la realidad como los otros sujetos del estudio la experimentan y desde ese escenario puedan interpretar y reinterpretar los hallazgos alcanzados.

Considerando aspectos de orden temporal y prácticos, se optó por trabajar con una muestra de nueve personas con los siguientes parámetros sociodemográficos claves para el estudio:

a. Que estuviesen inscritas en el Registro Nacional de Mediadores del Ministerio de Justicia chileno.

b. Que estén trabajando en mediación familiar durante el último período de 12 meses.

c. Que hayan sido evaluadas con la metodología Ecame.

d. Que se desempeñen en ciudades de la zona central de Chile.

Teniendo en cuenta la pregunta guía del estudio, que fue definida y ajustada hasta llegar a cuatro objetivos específicos, fue posible avanzar a la construcción operativa de las variables o ejes de análisis del estudio, que a continuación se detallan y que fueron abordadas teóricamente en el marco referencial que orientó el trabajo (véase tabla 1).

Tabla 1. Matriz de ejes de análisis

\begin{tabular}{|l|l|l|}
\hline \multicolumn{1}{|c|}{$\begin{array}{c}\text { ASPECTOS } \\
\text { TEÓRICOS }\end{array}$} & \multicolumn{1}{|c|}{ META ESPECIFICA } & \multicolumn{1}{c|}{ CATEGORía DE ANÁLLISIS } \\
\hline $\begin{array}{l}\text { Modelos y } \\
\text { sus etapas } \\
\text { de mediación } \\
\text { familiar }\end{array}$ & $\begin{array}{l}\text { Descripción de modelos } \\
\text { utilizados y los momentos } \\
\text { de trabajo que identifican } \\
\text { los mediadores en su trabajo } \\
\text { cotidiano, analizando la } \\
\text { aplicación de principios } \\
\text { dogmáticos. }\end{array}$ & $\begin{array}{l}\text { Modelos de mediación } \\
\text { utilizados frecuentemente } \\
\text { Tipos de causas en que se } \\
\text { aplican. } \\
\text { Aplicación de los principios en } \\
\text { la realidad chilena: alcances, } \\
\text { fortalezas y obstáculos. }\end{array}$ \\
\hline $\begin{array}{l}\text { Técnicas y } \\
\text { herramientas } \\
\text { usadas dentro } \\
\text { de la sala de } \\
\text { mediación. }\end{array}$ & $\begin{array}{l}\text { Análisis de la aplicabilidad de } \\
\text { las técnicas y herramientas de } \\
\text { la mediación, según tipología } \\
\text { de causas y contexto en que } \\
\text { trabajan. }\end{array}$ & $\begin{array}{l}\text { Descripción de las técnicas y } \\
\text { herramientas más utilizadas y } \\
\text { los productos obtenidos. } \\
\text { Apreciación personal de su } \\
\text { aplicabilidad en la realidad } \\
\text { chilena, según tipo de causa y } \\
\text { contexto que atienden. } \\
\text { Fortalezas y debilidades } \\
\text { personales detectadas para la } \\
\text { utilización de ciertas técnicas } \\
\text { sobre otras. }\end{array}$ \\
\hline
\end{tabular}

\begin{tabular}{|c|c|c|}
\hline $\begin{array}{l}\text { ASPECTOS } \\
\text { TEÓRICOS }\end{array}$ & META ESPECIFICA & CATEGORÍA DE ANÁLISIS \\
\hline $\begin{array}{l}\text { Resultados } \\
\text { alcanzados } \\
\text { en la sala de } \\
\text { mediación y } \\
\text { en la pauta } \\
\text { Ecame. }\end{array}$ & $\begin{array}{l}\text { Comparación del mediador de los } \\
\text { resultados que evalúa alcanzar } \\
\text { en la sala de mediación y los } \\
\text { resultados de la pauta Ecame. }\end{array}$ & $\begin{array}{l}\text { Reflexión acerca de los } \\
\text { resultados en los procesos de } \\
\text { mediación que realizan y las } \\
\text { razones de ello. } \\
\text { La relación que establece entre } \\
\text { sus resultados y la evaluación } \\
\text { Ecame: cercanía-distancia de } \\
\text { resultados. }\end{array}$ \\
\hline
\end{tabular}

Fuente: elaboración propia.

Se utilizaron fuentes secundarias de información (Flick, 2007), bibliografía de clásicos, documentos, investigaciones recientes y autores emergentes en las temáticas de la mediación, en general y en temas de familia, en particular, como también para el abordaje de la calidad de servicio desde el enfoque narrativo, lo que facilitó el tratamiento de la información.

La fase de campo se trabajó con la técnica primaria de entrevista semiestructurada aplicada a los sujetos de estudio, lo que permitió la construcción de los discursos y tipologías de terreno elaboradas especialmente para esta investigación.

El tratamiento de la información recolectada se realizó respetando los criterios de rigor científico necesarios para asegurar la validez y confiabilidad de los resultados alcanzados (Martínez, 2006). Para ello, se definió el uso de la estrategia de codificación y análisis de discurso, la que consiste en condensar el grueso de los datos en unidades analizables, creando categorías con ellos o a partir de ellos, que permitieron transformar los datos duros de la realidad en conceptos o grupos de conceptos susceptibles de ser analizados y relacionados con posterioridad.

Avanzando en el análisis de los datos para el discurso, fue posible trabajar en la perspectiva de crear vínculos y relaciones entre los conceptos y categorías construidas previamente, junto a las categorías emergentes de la propia realidad. Para profundizar en el análisis e interpretación de la información, se utilizó la metodología de triangulación (Martínez, 2006), privilegiando en esta oportunidad los criterios de consistencia, credibilidad y neutralidad. 


\section{Resultados alcanzados}

Mirando los modelos de mediación utilizados en la práctica de las y los mediadores familiares, se encontró que el paradigma constructivista y sistémico que subyace en este enfoque de trabajo profesional en la mediación familiar se encontraba ya recogido en el mensaje presidencial (1997), en el que el Poder Ejecutivo propone la creación e instalación de un sistema colaborativo para la resolución de los conflictos en temas de familia y que fue recogido en la Ley n. ${ }^{\circ} 19.968$ (2005), que rige el actual sistema nacional de mediación familiar. Y que se encontraría presente en los distintos modelos de mediación conocidos y estudiados en Chile, como son el Modelo de Mediación de Harvard (Ury, 2012), el Modelo Circular Narrativo Cobb y Suares (Cobb, 2011; Sluzki, 1998-2011; Suares, 2005-2012), el Modelo Transformativo (Bush \& Folger, 2006; Folger \& Ganope, 2008; Shailor, 2005) y el Modelo Eco-sistémico (Parkinson, 2005).

La práctica mediadora estudiada permitió entender, entonces, que la definición de mediación familiar que prima en el funcionamiento del sistema es aquella que pone la legalidad vigente, conforme a las definiciones que contiene el Título $\mathrm{V}$ de la Ley n. ${ }^{\circ} 19.968$, como los instructivos y reglamentos emanados del ente administrador que es la UMED. Se construye así una propuesta de mediación familiar vista como un proceso de resolución de conflictos regulado por un conjunto de procedimientos legales y administrativos, que son aplicados conforme a las necesidades y requerimientos del caso atendido, buscando la consecución de acuerdos y soluciones pragmáticas dentro de las condiciones de demandas y recursos que exhiben las personas involucradas en el conflicto abordado. Como consecuencia de esta conceptualización de la mediación, el trabajo de los y las mediadores, más que ser definido desde un enfoque teórico, pasa en la práctica a ser diseñado casuísticamente teniendo como escenario de fondo los principios y normas legales vigentes. Estos, por su amplitud y aplicación, permiten ser ajustados a los requerimientos de cada caso atendido.
Ese planteamiento de corte básico y práctico, mayoritariamente, deja fuera del trabajo por realizar por el mediador o mediadora la construcción de la hipótesis de trabajo del caso que se abordará, lo que se entiende necesario de enlazar al conocer el conflicto a tratar y que es un planteamiento transversal de todos los modelos teóricos de la mediación. La importancia de la hipótesis del caso (Donoso, 2010) se sustenta en que le permite al profesional comenzar a identificar las temáticas y relaciones que muestran las partes y son ejes del conflicto en particular y así diseñar de manera exploratoria la intervención que realizará. Así, surge entonces la inquietud de cómo se diseña una intervención profesional sustentable y rigurosa sin un diagnóstico del caso.

La ontología de la mediación familiar, igualmente, se resguardaría en los principios de voluntad de participación, equilibrio de poderes o igualdad y protagonismo de las partes; tres ejes rectores que ponen en el centro de cualquier proceso de gestión de conflictos (Sparvieri, 1996), la necesaria presencia y compromiso de las personas que afrontan el problema, como herramientas básicas para la resolución del mismo. Con ello se aporta a la autocomposición de las partes para la resolución de sus conflictos. Asimismo, se visualizan los principios de confidencialidad, imparcialidad y neutralidad en el desempeño del mediador o mediadora que es responsable de la atención del caso en cuestión. Se refuerza así la intervención profesional para la construcción de canales de comunicación que permitan una evaluación del conflicto tendiente a identificar posibles vías de resolución que satisfagan a los y las afectados (Garrido \& Munduate, 2014). Por ello, deben ser puestos en práctica durante toda la mediación y no solo al inicio como información del proceso, lo que es señalado como parte del ejercicio de las profesionales. De esto se desprende que hay una consideración al componente relacional del conflicto, pese a que no se desarrolle un diagnóstico al respecto.

La especial consideración al principio de interés superior del niño, niña y adolescente estaría enmarcada por la temática de familia y el 
miramiento de protección integral de los hijos e hijas (Hernández, s.f.), tanto en la existencia como en la resolución de la problemática en tratamiento. Lo anterior es coherente con la mirada del modelo ecosistémico (Parkinson, 2005), que declara explícitamente su aplicación a la problemática familiar y la importancia de relevar la figura de los hijos e hijas como actores del grupo familiar, que deben ser considerados de manera especial en el proceso de la mediación y en las soluciones, de corte parcial o final que se alcancen.

Desde esa perspectiva, se retoma la consideración de alcanzar un producto que mejore la comunicación entre las personas (Link, 2005), así como la comprensión del contenido del problema para la construcción de acuerdos que lo resuelvan de forma adecuada para los afectados. Por tal motivo, se valora con especial importancia la instalación de ciertas formas de funcionamiento colaborativo entre las personas y el mediador o mediadora, tales como el respeto entre los asistentes, no interrumpir, no presentar conductas de agresión o violencia que dificulten o contaminen el espacio imparcial y colaborativo diseñado para el desarrollo de la mediación. Y, en especial, la evaluación de posibles conductas de violencia intrafamiliar constitutivas de delitos o de corte complementario (M. Justicia, 2010; Rioseco, 1999), con el fin de evitar la exposición de las personas a estos episodios que por la agresividad que contienen no corresponden a esta intervención y no son posibles de ser atendidos en la sesión de mediación. Hasta este momento, llama la atención la especial consideración a aspectos de forma de las relaciones que se establecen entre los participantes de la mediación familiar, lo que debe entenderse en el contexto de las relaciones humanas y de comunicación que enmarcan la intimidad de las relaciones familiares.

La noción de la mediación familiar como un proceso de atención que dispone de diferentes momentos de trabajo, conforme el desenvolvimiento de la problemática, si bien no es declarada explícitamente, se entiende presente en el desarrollo de la atención mediadora propiamente tal, tanto en los momentos de inicio y presentación de la mediación, como en la gestión del problema, y en la búsqueda de soluciones y cierre de la atención (Fundación Libra, 1996). No obstante, haciendo nuevamente el vínculo con la ausencia de la hipótesis del caso, se mantiene la inquietud teórica de cómo se realiza la pesquisa de la problemática real del caso, avanzando en la diagramación de las posturas y expectativas a los intereses, necesidades y las creencias que los sustentan, para poder así definir, en primer lugar, la viabilidad de la atención en mediación y, luego, las estrategias y técnicas de intervención más acordes a las características del conflicto y de las personas que lo afrontan.

Los tipos de causas que son atendidas en la mediación familiar licitada corresponden a los definidos para mediación previa en la legislación vigente (Ley 20.260), como la pensión de alimentos, relación directa y regular y cuidado personal, los que, según la información recopilada, no serían atendidos de manera especial con un solo modelo teórico. Cuando se abordan las causas referidas a manutención de los hijos e hijas serían atendidas con mayor uso de herramientas propias del Modelo de Harvard, en consideración a los aspectos materiales, que permiten la generación de transacciones entre las partes, conforme a las peticiones y propuestas que intercambian los y las asistentes. En tanto que el tratamiento de las materias de relación directa y regular como de cuidado personal recurriría al uso de herramientas del Modelo Circular Narrativo (Sluzki, 2011; Suares, 2012) y del Modelo Transformativo (Bush \& Folger, 2006; Shailor, 2005), por el énfasis en el cuidado de las pautas comunicacionales y relaciones de los integrantes del grupo familiar, con una especial consideración al ambiente de intimidad y continuidad del vínculo familiar.

Todos los elementos señalados por los sujetos del estudio, que conforman la imagen de la mediación familiar desde los y las operadores que la aplican cotidianamente, se encuentran recogidos en su totalidad en la definición de la mediación familiar con enfoque de calidad que postula la UMED (PUCV, 2012). Esto permite concluir que hay coherencia conceptual básica entre la propuesta de 
un modelo de mediación familiar de calidad que estaría actualizando la UMED y la que han ido construyendo e interiorizando los y las mediadores familiares licitados. Lo anterior, sin embargo, al contrastarlo con los requerimientos que propugnan los modelos teóricos de la mediación, muestra una diferencia relevante en la definición del conflicto familiar y el abordaje científico del mismo. Cabe preguntarse ¿por qué se presenta esta situación y la vinculación que podría tener con la concentración de temáticas que son atendidas por los y las profesionales dentro del SNM?

Con esta primera consideración de la mediación y calidad, entre la construcción teórica del Estado y los operadores del sistema de mediación familiar licitada, es posible ahora valorar las herramientas y técnicas utilizadas que permiten esta coherencia. La consideración del contexto en que se presenta y las características de las personas en disputa son elementos claves que determinarían el trabajo y aplicación de metodologías que realizaría el profesional en la sala de mediación. De acuerdo con la práctica analizada, habría tres variables importantes para tener presentes respecto de las personas que son atendidas. Una de ellas dice relación con la edad de los y las participantes; en ésta, los sujetos que se encuentran bajo la barrera de los treinta o treinta y cinco años, serían más proclives desde el comienzo a trabajar en un proceso de mediación, mediatizado por ser parte de una generación más dispuesta al diálogo y la búsqueda de soluciones consensuadas.

La educación formal de las personas pasa a ser otro elemento para tener en cuenta por el mediador o mediadora en dos sentidos. La consideración de la educación formal, asociada a la comprensión del lenguaje de la mediación en un escenario judicializado, como un criterio que debe tenerse presente de modo de asegurar que las partes entiendan adecuadamente el proceso de mediación y los posibles acuerdos de solución al conflicto que alcancen. Y, también, que cuando hay mayor formación técnica y profesional asociada a mayores ingresos económicos de las partes, se observan mayores resistencias subjetivas en los sujetos de abordar aspectos relacionales para el afrontamiento integral del conflicto. Ambos casos le exigen al profesional desplegar una cantidad mayor de recursos destinados a la contención y tratamiento de la información disponible en la sesión. Asimismo, la situación económica y laboral de los y las usuarios fue otro elemento identificado desde las condiciones del entorno que afectarían el desarrollo de la mediación, asociado a la estabilidad de ingresos que favorecería la vinculación con los hijos e hijas.

La disponibilidad para asistir y el interés de las personas por acudir a segundas o más sesiones de mediación, ya que no dispondrían de permisos laborales para ello, pasa a ser un obstáculo recurrente en el actual estado de la mediación. Esta razón llevaría a optar por el trabajo de búsqueda de posibles acuerdos en una sesión única de mediación, lo que incidiría directamente en la profundidad del diagnóstico de caso y en la sustentabilidad de los acuerdos.

Cuando se abordó el tema de los modelos de la mediación que usarían los y las mediadores familiares, se comprobó que dentro de la mirada sistémica y constructivista que guía su accionar, la identificación del uso de uno u otro modelo la realizaban mayoritariamente con el uso de ciertas técnicas por sobre la mirada ontológica de gestión del conflicto. Esta mirada pragmática para diseñar y ejecutar el desarrollo de una mediación familiar se observó en la selección de herramientas más nombradas para realizar la sesión de trabajo.

Dentro de lo que se podría considerar el momento de inicio de la mediación, se mencionó el discurso inicial (Fundación Libra, 1996) como la técnica por excelencia que les permite explicar en qué consiste la mediación, sus principios, reglamentos y forma de trabajo, constituyendo en la práctica en el rayado de cancha que enmarcaría el trabajo colaborativo por desarrollar y al que recurrirían con éxito de manera recurrente.

Para la gestión del conflicto, mencionaron un amplio número de técnicas para el diagnóstico, gestión y reconstrucción de la narrativa del conflicto para la búsqueda de soluciones concretas y 
pragmáticas al problema recibido. Entre estas técnicas mencionadas están las de corte interrogativo, como las preguntas abiertas, preguntas cerradas, preguntas aclaratorias, que les permitirían conocer la narrativa actual de cada parte involucrada en el problema en gestión, así como las herramientas de corte afirmativo: parafraseo, resumen, encuadres y reencuadres que permiten ir precisando la temática que se abordaría en la mediación. Esto se evaluó de gran utilidad para poder precisar y devolver los puntos centrales de los relatos a las partes, de modo de poder ir sentando aspectos relevantes del proceso que luego podrán ser retomados en el trabajo reflexivo y de búsqueda de acuerdos.

Las herramientas destinadas a apoyar el empoderamiento de las partes, como son la connotación positiva y la revalorización, se mencionan desde el comienzo de la sesión de trabajo y durante el desarrollo de la misma. Su aplicación permanente siempre debería disponer de una base de realidad, generando credibilidad en la evaluación del mediador o mediadora. Esto plantearía un fuerte vínculo con mantener la imparcialidad de la figura del tercero o tercera neutral (Moore, 2005), mientras que, entre las partes el sentirse valoradas por un tercero o tercera, este pasaría a ser un recurso de fortalecimiento de su propio rol y que les permitiría comenzar a mirar los aportes disponibles en la otra parte, lo que ha sido buscado por el profesional como una contribución al trabajo de gestión colaborativo de conflictos.

Avanzando en la gestión del conflicto, se identifica siempre una amplia batería de preguntas como las ya indicadas, a las que se agregan las preguntas de corte reflexivo, circulares y recontextualizantes, que permitirían apoyar el proceso deliberativo de las partes involucradas y su rol en el conflicto (Suares, 2012). Estas preguntas tienen en común que son utilizadas para fomentar la evaluación personal que pueden realizar las personas sobre su vivencia y los efectos que tienen en sí mismas y en los otros cercanos, apoyando así la circularidad de la información y, con ello, la visualización de posibilidades diferentes de entender lo que está sucediendo y comenzar a pensar en distintas formas de resolución, por lo que son altamente valoradas en su aplicación cuando son acogidas por las partes. Esto debería hacer que el profesional se detenga en la evaluación de recursos y condiciones de los y las usuarios de la mediación, prestando especial atención a los aspectos ya identificados de edad, educación y nivel socioeconómico, de modo de apoyar efectivamente el proceso de análisis de la problemática en el contexto real que se desenvuelve.

Nuevamente se mencionan el uso de parafraseos, resúmenes y reencuadres, para afirmar las declaraciones y acuerdos que comienzan a generarse en la mediación, como también la aplicación de las herramientas destinadas al empoderamiento de las partes que ya fueron identificadas en los párrafos precedentes.

Tanto en la fase inicial como de gestión del conflicto, comienzan a aparecer las áreas del conflicto y que requieren de intervención de mayor complejidad y ser contrastadas con la hipótesis o diagnóstico inicial del caso en tratamiento. Esto no siempre es posible en el SNM, ya que no se requiere por normativa de una base teórica compleja que oriente la atención. Lo anterior hace surgir las siguientes preguntas: ¿cómo se ordena y prioriza el trabajo de guía y de facilitación que debe realizar el mediador o mediadora en su rol de conductor del proceso? Y, vinculado a ello, ¿de qué manera el mismo sistema, así como el profesional pueden, entonces, realizar la evaluación del trabajo realizado, de modo de valorar los aportes efectuados al tratamiento del caso y el mejoramiento de la situación de las partes, como los aprendizajes adquiridos producto del contacto con la realidad?

Para la etapa de cierre de la mediación, se privilegiaría el uso de técnicas como la lluvia de ideas, el abogado del diablo y agente de realidad (Ury, 2012), las que estarían destinadas a apoyar la identificación de otras formas posibles de resolver el problema compartido, que sean sustentables en el tiempo, conforme a los recursos y posibilidades de ambos involucrados. El concepto pragmático de sustentabilidad lo apoyarían en la aplicación de 
criterios externos y por ello objetivos, los que son aceptados por las partes. La metodología de aplicación es la contrastación de preguntas, de modo que los y las usuarios puedan identificar y valorar las dificultades, y los aportes que encontrarían en su implementación con el fin de cuantificar la viabilidad del mismo.

Las técnicas y herramientas señaladas por los y las mediadores fueron identificadas con los modelos de Harvard y Circular Narrativo mayoritariamente, aun cuando no detallan las diferencias que podrían tener en su utilización dependiendo de cuál modelo orienta o adscribe en su accionar. Además, su uso estaría orientado en la lógica pragmática de avanzar en la búsqueda de resultados para los acuerdos, tanto en el plano de los canales comunicacionales como en el foco de la postura y expectativas del problema que se abordó en la sesión, lo que es coherente con la definición operativa del modelo de mediación del SNM por sobre cualquier otro propuesto teórico. A estos modelos, corresponden aquellas herramientas que según los datos empíricos recopilados son utilizados adecuadamente por los y las mediadores, considerando los resultados que les aportarían en cuanto a favorecer las comunicaciones entre las personas en la sala de la mediación, así como en la búsqueda de acuerdos conforme a las peticiones, de tipo parcial o total.

Es curioso que el Modelo Transformativo no sea mencionado de forma específica como modelo orientador de la práctica mediadora, tal como fueron señalados otros dos modelos. Esto puede explicarse por la ausencia de las consideraciones de intereses y requerimientos de cambio actitudinales, que son parte de las consideraciones preliminares de la hipótesis y propuestas de intervención de los modelos en general, pero que son imprescindibles en el caso de la aplicación del Modelo Transformativo. El mismo análisis es aplicable a las herramientas propias de este modelo, como son la revalorización de sí mismo, el reconocimiento del otro y la concientización del cambio posible de desarrollar. Lo anterior se puede explicar solo considerando las debilidades detectadas por los operadores del sistema, que refieren la escasa aplicación de estas herramientas por no disponer de formación adecuada para su comprensión, aplicación y evaluación de los resultados alcanzados con ellas.

Este punto, al conectarlo con la importancia que le otorgan los y las mediadores a la capacitación continua y el cuidado en los temas de convivencia familiar y social que son atendidos, se muestra como una posibilidad de acción validada por los y las operadores que podría apoyar en fortalecer esta arista formativa detectada por el colectivo de mediadores, que se entiende enfocada desde el pragmatismo, para disponer de otras herramientas que les permitan avanzar en la consecución de acuerdos sustentables.

Contemplando la forma en que los y las mediadores realizarían el trabajo en la sala de mediación bajo los criterios de calidad definidos por la UMED (PuCv, 2012; Universidad de Chile, 2014), encontramos que se mantiene coherencia entre las prácticas declaradas y los criterios de evaluación del Estado. Ambos actores trabajan con la idea de la mediación como un proceso de atención a personas que tienen o vivencian un conflicto, que puede ser gestionado o abordado con un conjunto de herramientas y prácticas que promueve un externo mediador o mediadora, con la finalidad de buscar posibles soluciones novedosas a las manifestaciones del conflicto familiar que satisfagan a los integrantes del grupo familiar.

Es posible valorar que, para alcanzar esta propuesta de intervención, la práctica mediadora conocida en esta investigación considera relevante en todo el proceso de trabajo la existencia y aplicación de los principios rectores transversales a los modelos de mediación, y con especial atención los referidos a la protección integral de la niñez y juventud, así como las normas legales vigentes que sean transversales a todas las causas posibles de atender. Se da así cumplimiento a los puntos considerados en el Componente de Legitimidad (PUCV, 2012), que centra el foco en la presentación y aplicación de los ejes rectores y la normativa legal, en 
el trabajo del mediador durante el trabajo en la sala de atención.

Asimismo, pone especial atención al desarrollo de prácticas conductuales y actitudinales del mediador o mediadora destinadas a favorecer el modelamiento de nuevas pautas comunicacionales, colaborativas y respetuosas del otro u otra, que se muestran en un espacio especialmente preparado y controlado para ello (Bush \& Folger, 2006). Esto respondería al eje relacional del conflicto y es coincidente con el componente de comunicación considerado en la conceptualización del Ecame, que debe estar presente durante todo el trabajo de la mediación. Se considera que con ello es posible avanzar en la generación de espacios de fomento de la participación y reflexión pragmática respecto de la vivencia personal y sus efectos en terceros cercanos que apunten a entender de forma comprensiva la experiencia vivida, involucrando a las otras partes con quienes comparten el conflicto, en la identificación de soluciones comunes que sean factibles de alcanzar.

El foco del contenido de conflicto, tal como se señaló en párrafos anteriores, desde un corte práctico y que permite arribar a soluciones al estado actual del mismo, permite valorar la correspondencia de objetivos con los Componentes de Participación y Resolutivo (Ecame), que se centran en las modalidades de fortalecimiento dentro de la sesión, para el empoderamiento y equilibrio de poderes entre los sujetos para identificar y valorar posibles alternativas de resolución de las disputas que sean posibles y satisfactorias para los y las intervinientes. Ello pone nuevamente el objetivo de atención en el proceso de modelamiento y monitoreo de formas de comunicación y búsqueda de puntos comunes que puede realizar el mediador o mediadora con las personas en la sala de mediación.

Aparece así, como categoría necesaria de incorporar en este análisis, la relevancia de la figura del mediador o mediadora, sus funciones y prácticas profesionales como elementos sustantivos de ser considerados para la hilación entre el perfil profesional definido y la calidad del servicio esperado. Las competencias profesionales que se entienden presentes en la definición de perfil que presenta la UMED (M. Justicia \& VRA Consulting, 2014) y en los relatos analizados en este estudio serían:

a. Integridad del profesional, perseverancia, compromiso y responsabilidad en el ejercicio del rol de servicio público. Son elementos observables en el cumplimiento de los principios rectores de la mediación durante el desarrollo del trabajo.

b. Flexibilidad, dinamismo, autocontrol, tolerancia, manejo de crisis y capacidad analítica y síntesis. Habilidades propias de las profesiones que trabajan en relaciones de ayuda a terceros y terceras, como es el caso de la mediación que se ocupa de la gestión de conflictos en temas de familia. Estas se observarían en la relación comunicacional y de fomento a la participación activa de los participantes en la mediación.

c. Comunicación efectiva y empatía. Competencias actitudinales que aportan a potenciar la prestación de calidad en el servicio brindado a la comunidad, potenciado el mejor aprovechamiento de los recursos disponibles. Esto es requerido cotidianamente en la observación del quehacer en sala de mediación que ha sido el foco de esta investigación.

Todas las competencias profesionales detalladas en el párrafo precedente corresponden a aquellas que deberían ser desplegadas en la atención directa con los y las usuarias del SNM, lo que lleva a la conclusión de que este es el plano laboral que los y las mediadoras identifican como propio de su desempeño y que es posible de ser valorado en la calidad del servicio. Sin embargo, dentro de la definición del perfil del mediador y mediadora que propugna la UMED desde el año 2014, existe un conjunto amplio de al menos otras seis competencias que no están consideradas en la imagen del rol de mediador o mediadora familiar que han construido en la práctica los y las mediadores avalados con el sistema Ecame de calidad de la mediación familiar. Las competencias que no estarían consideradas serían de tipo funcional, acordes a profesiones de servicios estatales y de ayuda a la población, donde se espera que exista trabajo en equipos multidisciplinarios que potencien la actuación de cada profesional, desde el análisis y diagnóstico 
conjunto de la situación por intervenir, así como la articulación en redes interinstitucionales que colaboran con productos de atención entre sí, en una consideración igualitaria de aportes por parte de los y las involucradas.

Respecto de esos puntos, cabe la reflexión en relación a cuál es la relación de dependencia de los y las mediadores licitados con el Estado y los mecanismos de control asociados a ello, que permitan la exigencia de estos criterios por una parte, como también la consideración técnica de la importancia y viabilidad de estos criterios de inserción a equipos y redes de trabajo, cuando por definición el quehacer del mediador y mediadora familiar ha sido centrado en la atención directa a las personas en conflicto dentro de la sala de atención. Y las funciones de coordinación con otros servicios, centradas en las figuras de otros profesionales y técnicos que laboran en los centros de mediación, sin que existan instructivos de parte del órgano estatal que muestren la necesidad de modificaciones a los procedimientos actualmente en uso. Sería interesante poner esto en el tapete de la discusión, de modo de enriquecer el ejercicio profesional, en el que el trabajo interinstitucional y en redes se entiende de suyo necesario para avanzar en procesos de calidad de servicio.

Como categoría emergente asociada al punto de las competencias profesionales, se muestra la necesidad que detectan los y las mediadores de poder disponer de un programa de autocuidado y cuidado de equipos de mediación, de modo de disponer de especialistas autónomos y funcionales para la tarea de la mediación familiar.

Finalmente, al considerar el foco de atención en las evaluaciones que realizan los y las mediadores sobre su práctica laboral y los resultados exhibidos en el sistema de evaluación Ecame que ha sido implementado por el Estado, es posible arribar a dos grandes puntos de interés. El primero, es la consideración compartida de los y las profesionales de que el resultado de la mediación corresponde principalmente a la tarea desarrollada por las personas que asisten a la mediación, sus características personales, estado del conflicto y los recursos disponibles que les permitan evaluar o no la existencia de escenarios alternativos mejores al que están viviendo. Ello implica la consideración del rol del tercero o tercera imparcial, en que prima la labor de facilitación y modelamiento de pautas comunicacionales y de tratamiento de la disputa, manteniendo la convicción de que los resultados, cualesquiera que sean, son resorte de las personas que viven el problema, por lo que la forma de término que adopte la mediación será lo adecuado para el estado de desarrollo del afrontamiento del conflicto posible de ser efectuado por los intervinientes. Se considera muy importante relevar este resultado, ya que es un reconocimiento al rol de tercero y externo que tiene el mediador o mediadora. Y que, en los sistemas colaborativos de gestión de conflictos, y en particular en la mediación, la vivencia de la disputa, sus consecuencias y vías de término o continuidad son compromiso de las personas que lo afrontan y deben ser respetados en esto. Es posible observar esta valoración en los relatos analizados, tanto en los puntos referidos a términos con acta de acuerdo como a los cierres con certificado de mediación frustrada.

El segundo, se refiere a que los resultados de la autoevaluación cotidiana que realizarían los y las mediadores serían similares a los alcanzados en al menos una de las evaluaciones Ecame en que participaron. Esto permite reafirmar la existencia de criterios compartidos, entre los operadores y el órgano estatal de evaluación de desempeños de la mediación familiar, en torno al trabajo directo de atención a las partes en el espacio de la sala de mediación, centrados en la consecución de resultados de acuerdos que permitan la modificación de la situación actual de las manifestaciones del conflicto, según lo expresa la definición de calidad de servicio. Se destaca que aprecian positivamente la metodología de supervisión clínica con enfoque de pares utilizada en las dos últimas aplicaciones de la pauta Ecame, ya que les habría permitido contar con un espacio de reflexión de su propia práctica, al mismo tiempo que conocer en la experiencia los elementos que priorizaría la UMED para el desarrollo del trabajo de mediación familiar. 
Como aporte posible de este tipo de metodología evaluativa, se muestra en los actores involucrados -tal como lo indica la teoría- la consideración de sus efectos como un componente de una política de capacitación continua para el mejoramiento de la calidad del servicio licitado. Por esto, la mención de la capacitación continua, como categoría emergente dentro de los relatos analizados, se califica de relevancia, ya que abre un espacio requerido por los y las mediadores para el mejoramiento de su desempeño, en temas de mediación y de familia, como los ya señalados. Además de que podría ser considerado un facilitador para el nuevo modelo de bases técnicas que se ocuparán en los próximos años, y que postula la consideración de atenciones en mediación familiar que dispongan de más de una sesión.

\section{Algunas propuestas de mejoramiento}

Durante el desarrollo del trabajo teórico y de campo realizado, han ido surgiendo ciertos ejes claves de la mediación familiar, que han permitido la construcción de algunas propuestas generales, orientadas al mejoramiento y actualización de los procesos de mediación familiar licitada, los que se presentan a consideración de los y las lectores.

Respecto de la importancia de un modelo de mediación familiar a nivel nacional

La importancia de entender la mediación familiar como un proceso de trabajo destinado a apoyar el trabajo reflexivo de las personas que están afectadas por conflictos familiares susceptibles de ser judicializados se encuentra en la base de esta propuesta. Esta consideración permitiría proponer un modelo básico integrado que incluya, más allá de algunas herramientas específicas como el consentimiento informado, la imagen de un trabajo que contiene en sí mismo etapas diferenciadas que deben arrojar ciertos resultados específicos, que permitirán o no el paso a los siguientes momentos de trabajo.
En la fase inicial, además de los elementos de presentación actuales, se debería considerar la elaboración de una hipótesis diagnóstica que permita la construcción de una tentativa línea de intervención, que puede ser actualizada y reorientada conforme el desarrollo de los distintos procesos de gestión de contenido y relacionales que se desplieguen en el tratamiento del conflicto por las partes y el tercero o tercera imparcial.

La consideración de la mediación como un proceso de etapas sucesivas y complementarias en la atención permitiría que, de acuerdo al modelo seleccionado como el más adecuado por el mediador o mediadora para la atención del caso, se pueda identificar qué herramientas son más adecuadas para la consecución de los distintos hitos que requiere cada modelo de mediación en su aplicación exitosa, así como también las innovaciones que sean necesarias implementar. Ello, además, se entiende en coherencia con la indicación que regula las bases técnicas del proceso licitatorio de la mediación familiar para los próximos años, que postula la realización de mediaciones que dispongan de más de una sesión de trabajo, mostrando con ello un cambio sustantivo con el quehacer actual. Y al mismo, tiempo, incorporaría la posibilidad de trabajar modelando acuerdos parciales en los distintos momentos del servicio de mediación, tanto a nivel de contenido como del aspecto relacional del conflicto en tratamiento. Estas consideraciones en relación al concepto y aplicación de la mediación como un proceso de trabajo complejo deben incluir la referencia del tiempo como una variable de apoyo a la maduración de resultados posibles de ser alcanzados para la resolución del conflicto real que están afrontando.

Respecto de la aplicación de los principios y las normativas chilenas que se aplican a la mediación familiar

Los preceptos básicos que organizan la mediación familiar deben ser potenciados de manera coherente con las actuales y futuras normas y reglamentos del sistema de justicia chileno, manteniendo una necesaria coherencia interna. Esto 
implica que para la construcción de normas, reglamentos e instructivos, se debe tener en cuenta los cuatro criterios/principios que determinan a la mediación, como son la voluntad de participación de las personas y su progresivo empoderamiento en la acción y el desempeño profesional del mediador y mediadora de imparcialidad y confidencialidad respecto del trabajo realizado en el abordaje de las temáticas trabajadas. Para esto, se propone que ante la existencia de normas legales y procedimentales que puedan mostrar diferencias con el espíritu de la ley de justicia de familia de acercamiento y accesibilidad a las personas, se trabaje coordinadamente desde el Estado como actor central y con poder real ante y con los otros actores institucionales para que en los reglamentos se puedan construir los necesarios puentes de acercamiento, sin que deban ser las personas y usuarios del sistema, los que enfrenten la disparidad de criterios en torno a una temática en conflicto, que redunda en el alejamiento de la justicia a su situación en particular.

\section{Respecto de las temáticas que se atienden en mediación familiar}

Hoy en día, es sabido que las materias que son sujeto de mediación previa corresponden a aquellas que a mediados de la década pasada concentraban altos porcentajes de demanda de atención de los tribunales de justicia. Sin embargo, la práctica cotidiana en mediación familiar mostró las complejidades actuales de las materias que son atendidas, relevando la fuerte presencia de criterios como violencia intrafamiliar, dificultades relacionadas con distintos tipos de familia y la coexistencia de temas en crisis familiar. Por lo cual se postula como un criterio modernizador del actual órgano estatal ampliar la base de materias que pueden ser mediadas previamente y de forma gratuita, apoyando la incorporación de temáticas con situaciones basales, donde existan, por ejemplo, familia con padres de un solo sexo, padres de distintas nacionalidades, familias con adultos mayores como responsables de los niños y niñas, cuadros de relaciones simétricas y esporádicas de violencia entre los adultos responsables, procesos de revinculación de relaciones parento-filiales y/o de relaciones entre hermanos, por citar algunos factores propios de la sociedad postmoderna en que nos encontramos. Los beneficios de esta ampliación de las temáticas redundarían en dos aspectos relevantes del actual servicio licitado. Por un lado, la apertura a nuevas tipologías de causas aportaría a que fuese necesario para los y las mediadores crear nuevas formas de atención para incluir en la práctica actual de la mediación familiar, evitando con ello la rutinización de la atención a la población. Y, junto con ello, podría generar una oferta de atención que dé respuesta efectiva a las problemáticas actuales de la familia chilena, en una lógica de mayor integralidad en la prestación de servicios, simplificando los procesos de derivaciones por atenciones parceladas como sucede hoy en día cuando hay causas que incluyen problemáticas basales como las señaladas y que por ello deben ser derivadas al sistema judicial tradicional.

\section{Respecto del fortalecimiento de herramientas para la mediación familiar}

Este punto, que debe estar presente en cualquier política de mejoramiento de la calidad de los servicios que provee el Estado a la población, debe ser un eje de las políticas de perfeccionamiento del recurso humano que se desempeña bajo su alero. Sin embargo, es necesario que para ello se consideren previamente dos criterios que enmarcan al trabajo por realizar y que se enfocan a fortalecer de manera integral el desempeño profesional de los y las operadores del SNM.

En primer lugar, se sugiere que se fortalezca la formación en la temática de familias en crisis y sus actuales formas de estructura y funcionamiento en el contexto socioeconómico de los países latinos al alero de la interculturalidad existente, para ampliar la comprensión de los fenómenos emergentes de fragilidad y permanencia de los sistemas familiares. En segundo lugar, la inclusión de acciones formativas continuas que posibiliten los espacios reflexivos para el fortalecimiento de la imparcialidad, manejo de las transferencias y contratransferencias y subjetividades que surgen en los procesos de atención en relaciones de ayuda a terceros, de modo de fortalecer la aplicación de 
los ejes de la mediación, donde como se ha visto, la metodología de supervisión de pares del Ecame es un aporte a ello.

\section{Respecto del rol del mediador y mediadora familiar}

La clarificación de la conceptualización del rol y funciones que debe desempeñar el mediador y mediadora familiar se entiende necesario de profundizar y luego difundir ampliamente entre todos los operadores del SNM, de modo de homogeneizar los requerimientos del sistema como una política clara y de mediano plazo. Ello requiere la definición y priorización de los espacios y tiempos de trabajo que se deben dirigir a los distintos componentes de las tareas que componen el trabajo de un mediador y mediadora familiar, sean a nivel de sala de mediación -como lo identifican los y las mediadores al día de hoy o en otros ámbitos de trabajo-, como pareciera desprenderse del listado de habilidades que propone el actual perfil laboral presentado por el Ministerio de Justicia.

Con esta definición, se haría posible identificar cuáles serían las características de perfil laboral, de tipo genérico, funcional y específicos que se requiere sean cumplidas y en qué calidad de servicio para la confección de las evaluaciones y autoevaluaciones de desempeño, con enfoque de integralidad y pertinencia.

\section{Respecto del sistema de evaluaciones de calidad de la mediación familiar}

La existencia de un sistema integrado de evaluación de calidad de servicio se muestra como un factor de modernización de la política pública, ya que aporta en la construcción de estándares de atención a la población, como también en la entrega de incentivos a los servicios que se destacan en su funcionamiento. En este punto en particular, destacan algunas propuestas emanadas de los relatos de los y las mediadores y que se refieren a:

a. La construcción de un programa de evaluación de la calidad, asociado al rol y funciones del mediador y mediadora familiar, que incluya la construcción del sistema de ingreso, permanencia y salida de los y las profesionales al mismo.

b. La necesidad de incluir procesos complejos de evaluación de desempeños, diferenciados conforme a criterios de orden geográfico, acceso a capacitaciones y tipología de atenciones realizadas, que permitan el fortalecimiento de la prestación de servicios, de acuerdo con las particularidades que puedan presentar las distintas zonas geográficas a nivel nacional.

La importancia de la evaluación de calidad de los servicios de la mediación familiar, producida de manera integrada y sistemática, sin duda que constituirá un gran aporte a la profesionalización de la mediación familiar, y de la mediación en general, aportando en la construcción de un paradigma de política pública de mayor paz social.

\section{A modo de cierre}

El estudio realizado cumplió el objetivo de analizar la correspondencia entre los modelos y las técnicas que aplican los y las mediadores familiares en el proceso de la mediación en Chile que son considerados exitosos y la metodología de calidad de la mediación familiar, pudiendo con ello dar respuesta a la pregunta respecto de las técnicas y modelos de la mediación que utilizan los y las mediadores familiares en Chile, que les permiten gestionar con éxito en conjunto con las personas los conflictos que se abordan. Además, permitió conocer los temas emergentes que son de interés para los y las mediadores familiares y la generación de algunas ideas de propuestas de mejoramiento al SNM, desde la mirada de los y las operadores del sistema.

\section{Referencias}

Baruch, R., \& Folger, J. (2006). La promesa de la mediación. Cómo afrontar el conflicto mediante la revalorización y reconocimiento. Buenos Aires: Editorial Granica.

Baruch, R., \& Ganon Pope, S. (2008). La mediación transformativa: un cambio en la calidad de la interacción en los conflictos familiares. Revista de Mediación, 1(2). Recuperado de http:// 
imotiva.es/wp-content/uploads/2013/06/ Revista_Mediacion_02_03.pdf.

Biblioteca del Congreso Nacional de Chile. (2004). Mensaje Presidencial. En Historia de la Ley $N^{\circ}$ 19.968. Recuperado de www.leychile. cl/Navegar/scripts/obtienearchivo?id...3/.../ HL19968

Cobb, S. (2011).Espacios liminares en losprocesos de negociación. Atravesando umbrales interpretativos y relacionales en una negociación en empresa familiar. Material para asistentes al Programa Internacional en Mediación Familiar. Buenos Aires.

Donoso, M., et al. (2010). Mediación Familiar. Un enfoque integral de la familia y su contexto legal. Santiago: Editorial UCHS.

Fisher, R., William, U., \& Patton, B. (2012). Si [...] de acuerdo. Buenos Aires: Editorial Norma.

Flick Uwe. (2007). Introducción a la investigación cualitativa. Madrid: Moratas.

Fundación Libra. (1996). Mediación, Introducción $y$ entrenamiento. Jornada de Capacitación en técnicas y destrezas en Mediación. Santiago: Editorial CPU.

Garrido, S., \& Munduate, P. (2014). Contra la neutralidad, ética y estética en el modelo circular narrativo de Mediación de Conflictos. Revista Telemática de Filosofía del Derecho (17), 139-166. Recuperado de http://eprints.ucm. es/27538/

Gergen, K. (1996). Realidades y relaciones. Aproximaciones a la construcción social. Barcelona: Paidós.

Gergen, K. (2008). Hacia un vocabulario para el diálogo transformador. En D. Fried Schnitman (comp.), Nuevos paradigmas en la resolución de conflictos. Perspectivas y prácticas (pp. 4372). Buenos Aires: Editorial Granica.

Hernández, M. (2009). La mediación familiar como perspectiva de garantía para el interés superior del niño/a en conflictos derivados del ejercicio de la patria potestad. Contribuciones a las Ciencias Sociales. Recuperado de www.eumedumed.net/rev/ccss/06/mhp.htm

República de Chile. (2004). Ley 19968: crea los Tribunales de Familia. Recuperado de http:// www.leychile.cl.
República de Chile. (2007). Ley 20286: modifica la Ley 19968. Recuperado de http://www. leychile.cl

Link, D. (1999). Mediación y Comunicación. En J. Gottheil y A. Schifrin, Mediación, una transformación en la cultura (pp. 135-152). Buenos Aires: Paidós.

Martínez, M. (2006). Validez y confiabilidad en la metodología cualitativa, Revista Paradigma, 27(2). Recuperado de http:// www.scielo.org.ve/scielo.php?pid =s 1011 $22512006000200002 \&$ script $=$ sci_arttext

Ministerio de Justicia. (2010). Informe de Criterios de actuación por parte de los centros de mediación contratados por el Ministerio de Justicia en materia de violencia intrafamiliar. Santiago: Sistema Nacional de Mediación, Nueva Justicia de Familia / Gobierno de Chile: Ministerior de Justicia, División Judicial, Unidad de Mediación. Recuperado de http:// www.mediacionchile.cl/pagina/wp-content/ uploads/2014/12/DOC-61.pdf

Ministerio de Justicia \& VRA Consulting. (2014). Competencias laborales Santiago de Chile. Recuperado de http://www.mediacionchile. cl/pagina/wp-content/uploads/2014/12/ DOC-.pdf

Moore, C. (2006). Elproceso de mediación. Métodos prácticos para la resolución de conflictos. Buenos Aires: Editorial Granica.

Parkinson, L. (2005). Mediación Familiar. Teoría y práctica: principios y estrategias operativas. Buenos Aires: Gedisa.

Pignouli, S. (2015). El programa sociológico de Niklas Luhman y su contexto. Revista Mexicana de Sociología, 77(2). Recuperado de http://www.revistas.unam.mx/index.php/ $\mathrm{rms} /$ article/view/48247

PUCV-CEAL. (2012). Estudio sobre la calidad de la mediación familiar en Chile. Valparaíso, Santiago. Recuperado de www.mediacionchile.cl

Rioseco, L. (1999). Género y derecho: Mediación en casos de violencia doméstica. Santiago: Instituto de la Mujer.

Shailor, J. (2005). Desarrollo de un enfoque transformador para la mediación. Consideraciones teóricas y prácticas. En D. Fried Schnitman, 
Nuevos paradigmas en la resolución de conflictos. Perspectivas y prácticas (pp. 185-206). Buenos Aires: Editorial Granica.

Sluzki, C. (Trad.). (1998). Atractores extraños y la transformación de las narrativas. Estados Unidos: The Handbook of Construtive Therapies.

Sluzki, C. (2011). El camino desde el conflicto a la reconciliación. La coexistencia como proceso evolutivo. Material para asistentes al Programa Internacional en Mediación Familiar. Buenos Aires.

Sparvieri, E. (1996). El divorcio. Conflicto y comunicación en el marco de la mediación. Buenos Aires: Biblos.

Suares, M. (2012). Mediación; conducción de disputas, comunicación y técnicas. Buenos Aires: Paidós.
Suares, M. (2005). Mediando en sistemas familiares. Buenos Aires: Paidós.

Taylor, S., \& Bodgman, R. (1986). Introducción a los métodos cualitativos de investigación. Barcelona: Paidós.

Universidad de Chile - Centro de Estudios Públicos de la Facultad de Ingeniería Industrial. (2014). Auditoría al Modelo de Contratación de Servicios de Mediación Familiar. Santiago de Chile. Recuperado de http://www. mediacionchile.cl/pagina/wp-content/ uploads/2014/12/Informe-Final-U-Chile.pdf

Ury, W. (2012). Supere el no. Buenos Aires: Editorial Norma. 